Ann. Biol. anim. Bioch. Biophys., I976, 16 (I), 85-95.

\title{
ÉTUDE CHEZ LE LAPIN DU MÉTABOLISME DES ACIDES AMINÉS DANS LA PAROI D'UNE POCHE CECALE ISOLÉE
}

\author{
Josette MARTY, Marie-Anne LAVARDE et P. RAYNAUD \\ Laboratoire de Physiologie de la Digestion et de la Nutrition, \\ Institut de Physiologie, \\ 2, rue François-Magendie, \\ 31400 Toulouse \\ Institut Catholique, \\ Laboratoive de Biologie, \\ 31, rue de la Fonderie, \\ 31068 Toulouse
}

\section{RÉSUMÉ}

Une technique chirurgicale permet de réaliser une poche avec la partie distale du cæcum, celle-ci étant isolée de la partie proximale qui reste en communication avec le tube digestif.

L'étude des variations de certains composants du sang avant et après irrigation des tissus pariétaux permet de tester le fonctionnement physiologique du cæcum opéré. La concentration et la répartition des acides aminés totaux fournissent un aspect global de la composition en substances azotées, tandis que les variations des acides aminés libres éclairent le métabolisme intermédiaire protéique. L'homogénéité des valeurs et la cohérence des variations permettent de considérer la poche cæcale comme un organe ayant conservé un fonctionnement physiologique. Elle paraît, en outre, douée d'une intense activité protéosynthétique.

\section{INTRODUCTION}

Les travaux de ces dernières années concernant la digestion chez le Irapin, attribuent un rôle important à la partie postérieure du tube digestif. Il se développe, en effet, dans le cæcum, une microflore riche en azote (GRIFFiTHs et Davies, I963) (Proto et Cioffi, I973) et dont l'activité métabolique est à l'origine d'acides carboxyliques (MARTy, RAYNaUd et Carles, I973).

Dans le contenu colique, Bonnafous après avoir constaté des variations de la teneur en azote, met en évidence un facteur lysant (I968) agissant sur les micro- 
organismes, et suppose une absorption des produits de résolution au niveau du côlon (r970).

L'absorption à travers la paroi digestive du cæcum et du côlon a été confirmée pour les acides volatils qui se trouvent à des concentrations élevées dans le sang efférent de ces organes : (Beauville, Raynaud et Vernay, ig74) (McMrlian, Edwards et PARKer, I975). Cependant, les recherches faites dans ce même sang veineux ne mettent pas en évidence une absorption d'acides aminés (MARTY J., I973). Quant aux acides carboxyliques fixes (MARTY et CARLES, I973), nous avons été amenés à penser qu'ils étaient utilisés et transformés dans le métabolisme pariétal.

Pour accéder directement à la paroi, ce qui sur l'animal vivant présente des difficultés expérimentales certaines, nous avons confectionné une poche sur le cæcum. Notre travail a consisté à étudier comment le fait d'isoler une partie du cæcum de l'ensemble de l'organe modifie le métabolisme pariétal et, en particulier, celui des acides aminés.

\section{MATÉRIEL ET MÉTHODES}

\section{Les animaux}

Avant d'être opérés, les lapins, d'un poids moyen de $3 \mathrm{~kg}$, ont séjourné au moins $3^{\circ}$ jours dans l'animalerie du laboratoire où ils reçoivent une alimentation constituée uniquement d'avoine, de luzerne et d'eau. Après l'opération, les lapins sont replacés dans les mêmes conditions. Pendant la totalité du séjour, chaque animal est pesé régulièrement en vue de l'établissement de sa courbe de croissance.

\section{La poche cacale}

La technique que nous pratiquons permet la confection d'une poche isolée dans un temps relativement bref. Le principe consiste à diviser le cæcum en deux parties d'importance équivalente, l'intégrité du système artério-veineux étant rigoureusement respectée.

\section{Technique opératoive.}

Les animaux sont anesthésiés au nembutal à raison de $30 \mathrm{mg}$ par kilo corporel. L'animal endormi est placé en décubitus dorsal, les membres en extension. Les poils sont rasés sur toute la région abdominale. Après laparotomie, le long de la ligne blanche, le cæcum est extériorisé sur un champ de gaze placé autour de l'ouverture. Une section transversale est pratiquée au niveau du dixième anneau. La moitié proximale, attenante au tube digestif, est refermée par une suture en bourse, suivie d'un surjet qui assure une parfaite étanchéité. Elle constitue le cæcum résiduel ou " cæcum témoin " qui nous servira de référence. La moitié distale constitue la poche cæcale. Elle est vidée du matériel digestif qu'elle contient et soigneusement lavée à l'eau tiède. Avant d'être refermée, cette poche ainsi isolée du tube digestif est munie de deux canules abouchées à la peau, l'une près de l'appendice cæcal, l'autre près de la suture. Ces canules permettront d'établir une circulation du liquide de perfusion.

Après l'opération, l'animal subit une perte pondérale de l'ordre du dixième de son poids initial. Dès le cinquième jour, l'animal opéré s'alimente normalement et la reprise de poids est effective, mais il faut environ un mois pour que l'animal retrouve son poids de départ. Par la suite, la croissance est du même ordre de grandeur que chez les lapins normaux de poids identique (CATAla, 1972).

La poche évolue avec le temps. Sa lumière se rétrécit et contient une substance épaisse qui paraît être du mucus desséché. Des lavages quotidiens ont pour but d'hydrater la paroi, d'éviter le développement d'une microflore parasite et l'accumulation des substances susceptibles d'être sécrétées par la paroi. Ces considérations nous ont fait estimer la période la plus favorable à l'expérimentation aux alentours du quinzième jour consécutif à l'opération. 


\section{Dosages biochimiques}

Les échantillons destinés à l'analyse quantitative des acides aminés sont soumis au préalable à différents traitements de purification.

\section{- Les acides aminés libres.}

Les acides aminés du plasma sont recherchés dans le surnageant après défécation par l'acide sulfosalicylique à raison de $50 \mathrm{mg}$ par $\mathrm{ml}$ de plasma.

Ceux du liquide de perfusion sont dosés soit après filtration sous vide sur verre frité et concentration du filtrat, soit après une double extraction alcoolique puis aqueuse. L'extraction alcoolique est effectuée par un certain volume d'alcool à $75^{\circ}$ porté à ébullition sous réfrigérant à reflux. Après filtration, le résidu de cette première extraction est repris par un volume égal d'eau et soumis à une deuxième extraction.

Les acides aminés libres des tissus pariétaux sont extraits selon le même procédé.

\section{- Les acides aminés totaux.}

L'hydrolyse des protéines des tissus pariétaux et du mucus recueilli dans la poche est effectuée en tube scellé par $\mathrm{HCl} 6 \mathrm{~N}$ à $120^{\circ}$ pendant 6 heures.

Le dosage des acides aminés est ensuite effectué sur autoanalyseur selon la technique de Carles et Abravanel (1970).

Les autres substances, en particulier le sodium, le potassium et l'urée, sont dosées directement dans le sérum sur Technicon SMA-I 2 selon les procédés définis dans Technicon Symposia $(1967)$.

\section{Expression des résultats}

- Les valeurs présentées dans les tableaux sont des moyennes obtenues à partir de lots de 5 à ro animaux et dont le nombre exact est d'ailleurs précisé dans la légende. La répartition de ces valeurs autour de la moyenne est exprimée par l'écart à la moyenne au seuil de probabilité $\mathbf{P}^{0,05}$.

- Quand il est nécessaire de comparer deux séries de valeurs, nous employons l'analyse de variance. La valeur de $\mathrm{F}$ est alors indiquée dans le tableau.

\section{RÉSULTATS EXPÉRIMENTAUX}

Nos investigations concernent certains composants du milieu intérieur, les acides aminés du sang, de la paroi digestive et de la cavité de la poche.

\section{Le milieu intérieur}

Par rapport au sang artériel on observe régulièrement (tabl. I) dans les deux sangs veineux, une légère baisse du phosphore et de l'urée sanguine (différence significative au seuil $\mathbf{P}^{\mathbf{0}}{ }^{05}$ ). Parmi les acides aminés (tabl. 2), 5 d'entre eux sont l'objet d'une diminution systématique et significative. Ce sont 1'isoleucine, la leucine, la phénylalanine, la tyrosine et la valine. Toujours par rapport au sang artériel, nous avons observé une légère augmentation systématique, mais non significative au seuil $\mathrm{P}^{0,05} \mathrm{du}$ calcium pout le cæcum témoin et une augmentation hautement significative des protéines sériques totales pour la poche.

On retrouve pour le cæcum témoin un des mécanismes déjà décrits à propos d'une étude in vivo de l'aminoacidémie, réalisée sur le Lapin normal (MARTY, I973). En effet, l'étalement des valeurs des concentrations exprimé par l'écart à la moyenne 


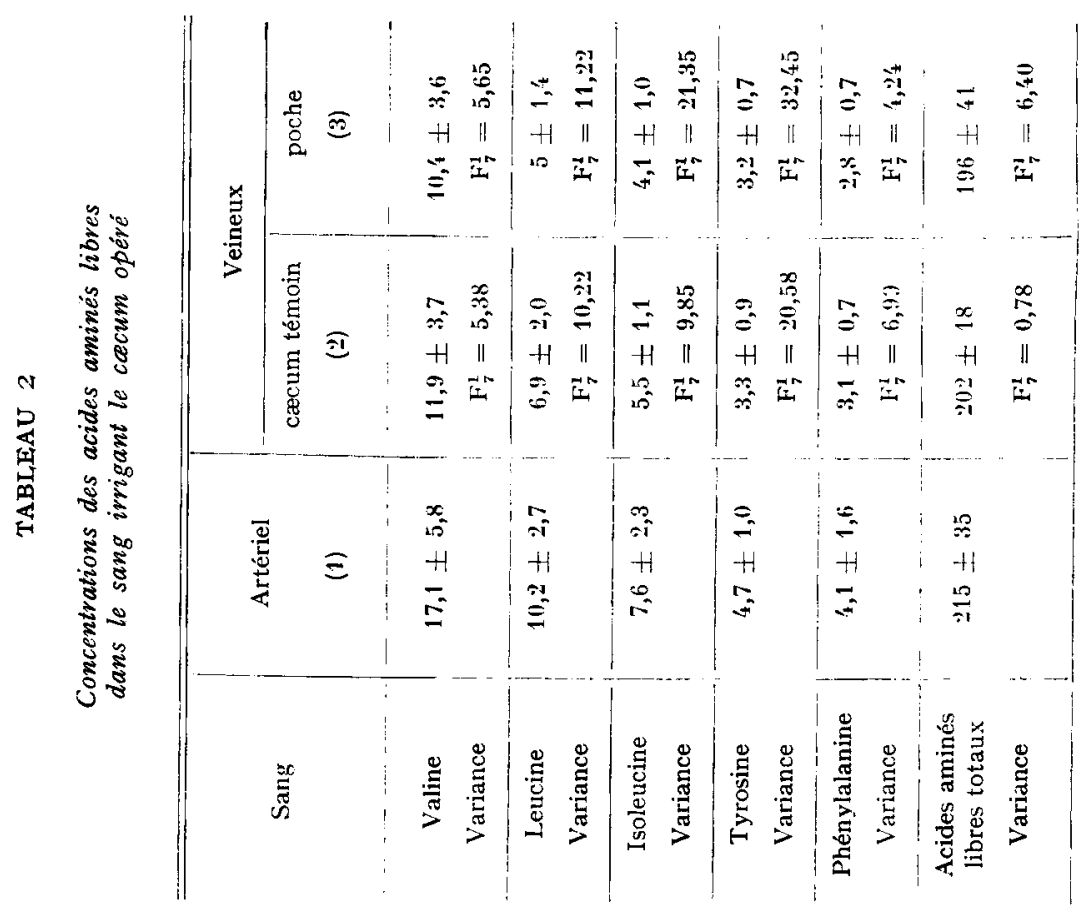

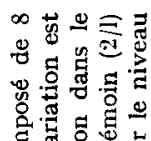

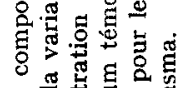

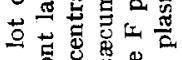

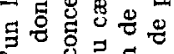

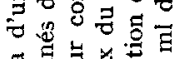

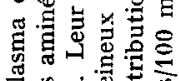

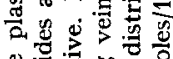

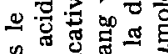

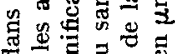

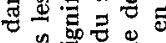

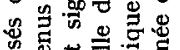

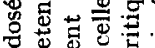

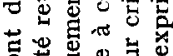

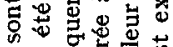
s

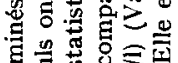
क जै की

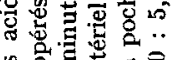

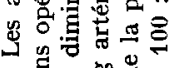

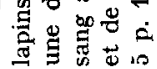

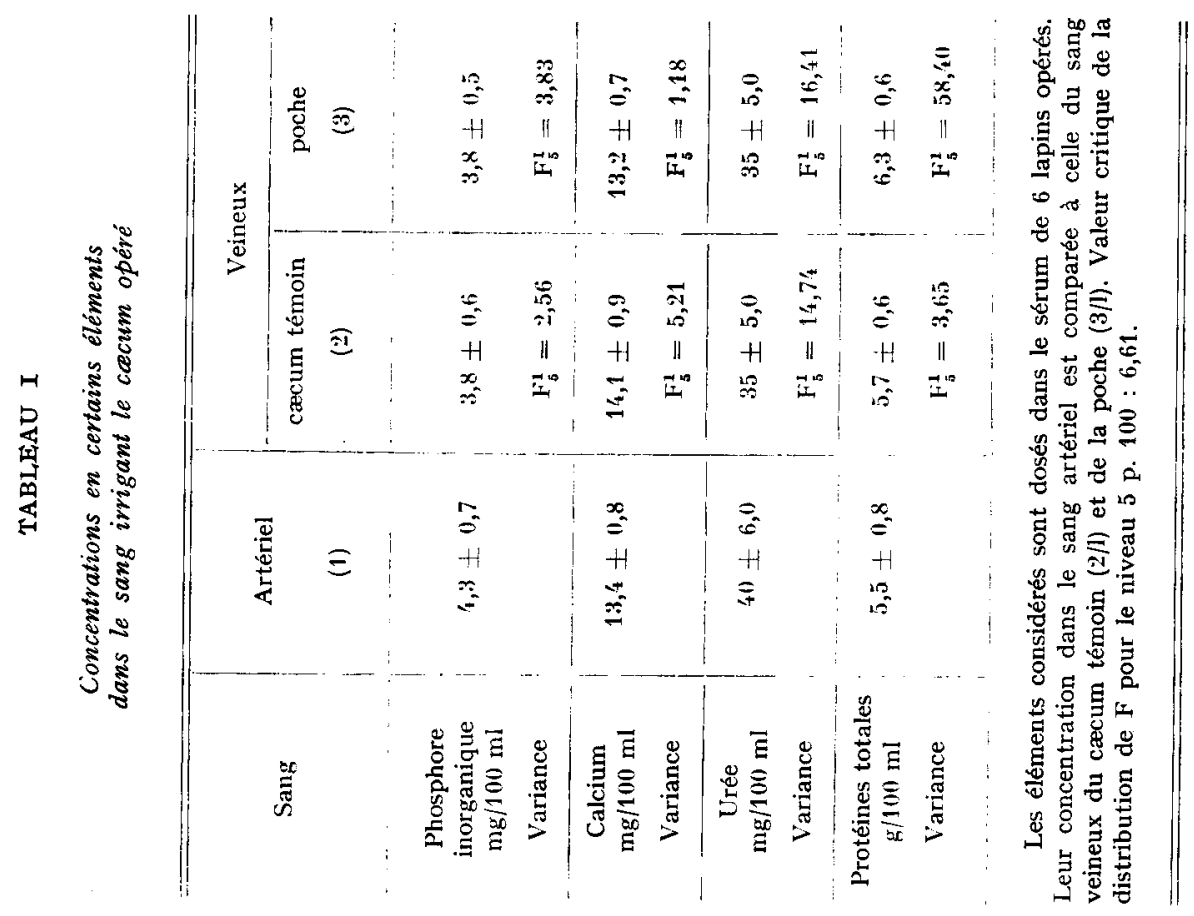


dans le tableau 2 a diminué après traversée de la paroi. Il n'en est plus ainsi dans le sang veineux de la poche où la teneur en acides aminés libres est égale ou le plus souvent inférieure à celle du sang artériel.

$\mathrm{La}$ comparaison statistique des concentrations ne montre pas de différence significative entre le sang veineux issu du cæecum témoin et le sang veineux issu de la poche cæale, exception faite pour les protéines sériques et l'isoleucine.

\section{Les acides aminés totaux.}

\section{La paroi cacale}

$\mathrm{L}_{\mathrm{a}}$ teneur en acides aminés totaux de Ioo grammes de paroi cæcale fraîche est de l'ordre de 40 millimoles pour le cæcum proximal des lapins normaux, de 44 millimoles pour le cæcum témoin des lapins opérés. Elle est de 56 millimoles pour le cæcum distal du lapin normal et pour la poche du lapin opéré.

\section{TABLEAU 3}

Étude des variations velatives des teneurs en acides aminés totaux de la paroi cacale

\begin{tabular}{|c|c|c|c|c|}
\hline \multicolumn{2}{|c|}{ Cæcum proximal } & & \multicolumn{2}{|c|}{ Cæcum distal } \\
\hline $\begin{array}{l}\text { normal } \\
(39,90)\end{array}$ & $\begin{array}{l}\text { opéré } \\
\text { "témoin " } \\
(44,35)\end{array}$ & & $\begin{array}{l}\text { normal } \\
\langle 55,55\rangle\end{array}$ & $\begin{array}{c}\text { opéré } \\
\text { " poche " } \\
(56,10)\end{array}$ \\
\hline 13,4 & 12,8 & Glu & 11,8 & 10,0 \\
\hline 6,8 & 5,4 & Lys & 6,6 & 6,1 \\
\hline 1,7 & 0,9 & Met & 1,6 & 1,3 \\
\hline 4,9 & 4,3 & Ser & 5,8 & 4,1 \\
\hline 2,5 & 1,8 & Tyr & 2,3 & 1,7 \\
\hline 4,5 & 5,0 & Ile & 4,1 & 5,0 \\
\hline 7,3 & 8,6 & Leu & 7,3 & 7,7 \\
\hline 6,8 & 7,7 & Val & 6,7 & 7,7 \\
\hline 5,0 & 4,5 & Arg & 4,8 & 5,0 \\
\hline 9,8 & 9,0 & Asp & 9,0 & 9,6 \\
\hline 11,3 & 11,5 & Gly & 12,6 & $13, k$ \\
\hline 3,5 & 3,5 & Phe & 3,3 & 3,5 \\
\hline 10,1 & 12,0 & Ala & 11,8 & 11,8 \\
\hline 6,6 & 7,4 & Pro & 5,6 & 6,2 \\
\hline 1,8 & 1,7 & His & 1,6 & 1,6 \\
\hline 1 & 3,9 & Thr & 4,1 & 5,3 \\
\hline 100 & 100 & & 100 & 100 \\
\hline
\end{tabular}

Les valeurs présentées dans le tableau sont les proportions relatives, exprimées en p. 100, des concentrations moyennes en acides aminés totaux d'une série d'hydrolyses effectuées sur deux lapins témoins et trois lapins opérés. Les acides aminés sont regroupés en fonction du sens de leur variation.

Le nombre réduit d'analyses est justifié par l'homogénéité des chromatogrammes représentatifs des parties considérées. La concentration des acides aminés totaux exprimée en millimoles pour $100 \mathrm{~g}$ de matière frấche est donnée entre parenthèses. 
- L'étude des proportions relatives (tabl. 3) nous renseigne sur les variations de la composition en acides aminés, indépendamment de fluctuations éventuelles de la teneur en eau. Elle montre qu'il existe chez l'animal des différences entre le cæcum proximal et le cæcum distal. Dans le cæcum proximal, la proportion des acides aspartique et glutamique est plus élevée que dans le cæcum distal. Par contre, l'alanine, le glycocolle, la sérine sont en proportions moins élevées. L,es autres acides aminés sont en proportions sensiblement équivalentes. L'opération renforce les différences pour l'acide glutamique et le glycocolle. Pour les autres acides aminés, la variation est nulle ou inverse.

- Si l'on considère maintenant l'effet de l'opération sur chacune des deux parties, on constate une augmentation commune des proportions relatives de l'isoleucine, de la leucine, de la valine, une diminution de l'acide glutamique, de la lysine, de la méthionine, de la sérine et de la tyrosine.

Les acides aminés libres.

Les concentrations des acides aminés libres $(798,6 \mu$ moles dans Ioo $g$ de matière fraîche) sont plus élevées dans les tissus de la poche que dans le cæcum témoin $(738,4$ $\mu$ moles dans Ioo g de matière fraîche). Cette différence est due essentiellement (tabl. 4) à une accumulation dans la poche de certains acides aminés : acides $\alpha$ et $\gamma$ aminobutyrique, acide glutamique, proline, citrulline, acide aspartique, alanine, glycocolle. Pour tous les autres acides aminés, on constate une diminution de la concentration et de l'importance relative. La valine est l'acide aminé le plus affecté.

\section{La cavité de la poche}

Le liquide qui a circulé en perfusion dans la cavité de la poche s'enrichit en acides aminés libres et en filaments de mucus plus ou moins abondants.

Les acides aminés totaux du mucus.

La répartition des acides aminés du mucus recueilli dans le liquide de perfusion et hydrolysé présente certaines fluctuations d'une expérience à l'autre (tabl. 5), mais les proportions de la leucine, de la valine et de la thréonine sont toujours parmi les plus élevées. D'un point de vue quantitatif, les acides aminés ne comptent que pour une faible part dans la composition biochimique du mucus, puisque nous trouvons des valeurs de l'ordre de 350 umoles d'acides aminés totaux dans roo $\mathrm{g}$ de mucus desséché.

\section{Les acides aminés libres.}

Les quantités d'acides aninés recueillies en perfusion sont faibles puisque les valeurs extrêmes sont 3 et 24 umoles dans nos expériences, et leur répartition totalement différente de celle trouvée dans un cæcum normal (MAR'Ty et CARLES, I968).

Après filtration du liquide de perfusion, l'analyse montre que $60 \mathrm{p}$. Ioo de l'ensemble des acides aminés est représenté en proportions sensiblement équivalentes par l'alanine (I 2,2 p. IOO), l'acide glutamique (II,4 p. IOo) la proline (I4,5 p. IOO) et le glycocolle (i I,9 p. IOo) et la sérine (II,7 p. IOo).

L'extraction alcoolique modifie cette répartition en élevant les proportions d'alanine $(\mathrm{I} 3,5)$, d'ornithine $(\mathrm{I} 5,5 \mathrm{p}$. IOo), de leucine $(\mathbf{I}, 6 \mathrm{p}$. IOo) et de valine (I3 p. IOO). 
MÉTABOLISME AZOTÉ, DE LA PAROI CAECALE
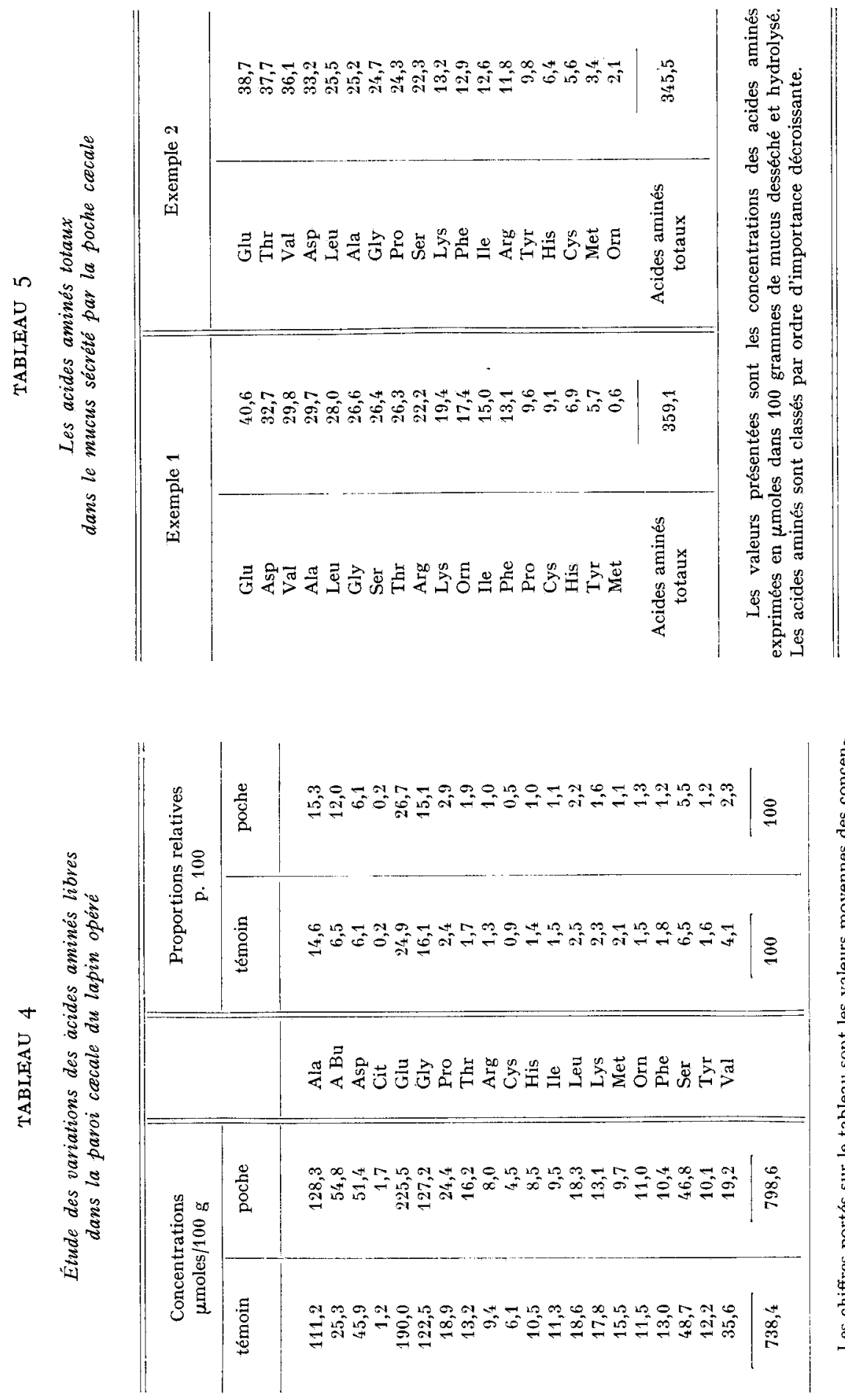


\section{DISCUSSION}

De l'étude biochimique réalisée dans le sang et les tissus pariétaux, il ressort que la physiologie de la poche cæcale ne présente pas un fonctionnement pathologique. Nous avons réuni à ce sujet un certain nombre d'arguments expérimentaux :

- la constance, après irrigation de la poche de certains éléments du sang tels le calcium; elle montre l'absence d'une concentration due à une déshydratation localisée dans la paroi de la poche;

- la similitude des variations entre les sangs veineux issus de la poche et du cæcum témoin par rapport au sang artériel (phosphore inorganique, urée, certains acides aminés signalés dans le tableau 2);

- l'identité des teneurs en acides aminés totaux dans la poche, et dans le cacum distal des lapins non opérés (tabl. 3);

- la réaction commune à l'opération en ce qui concerne les variations de composition en acides aminés totaux des deux parties opérées : augmentation relative des teneurs de certains acides aminés : isoleucine, leucine, valine compensée par une diminution de l'acide glutamique, de la méthionine, de la lysine, de la sérine et de la tyrosine;

- la présence de mucus dans la lumière de la poche qui suppose une activité sécrétoire de la paroi.

Il existe cependant, notamment pour certains acides aminés, des différences, mais leurs concentrations, chez le lapin opéré, restent du même ordre de grandeur que chez le lapin non opéré et nous considèrerons leurs variations en recherchant plutôt une signification dans les mécanismes métaboliques.

\section{La cicatrisation}

Il est certain que l'inflammation due à l'opération effectuée sur le cæcum, provoque une réaction des tissus. Dans les processus de cicatrisation interviennent les acides aminés soufrés et les acides aminés aromatiques. Leur mobilisation peut expliquer la diminution de la méthionine, de la phénylalanine et de la tyrosine dans le sang, et même dans les tissus des deux parties du cæcum opéré.

\section{La synthèse des substances mucoïdes}

- Le mucus prélevé dans la poche (tabl. 5) possède une composition en acides aminés assez variable, mais les proportions de valine et de leucine sont toujours très élevées. Pour que leur utilisation justifie les baisses observées dans les tissus pariétaux et dans le milieu intérieur, il faudrait que de grandes quantités de mucus soient sécrétées. D'autre part, cette sécrétion devrait être exaltée par l'opération et intensifiée dans la poche. Or, la partie azotée associée aux mucines, ne représente qu'une faible partie de la substance sèche. De plus, une simple extraction alcoolique (tab1. 6) paraît suffisante pour détacher des acides aminés de la structure mucoïde. 
TABLEAU 6

Acides aminés libres recueillis par perfusion de la poche cacale

\begin{tabular}{|c|c|c|c|}
\hline \multicolumn{2}{|c|}{ Avec extraction alcoolique } & \multicolumn{2}{|c|}{ Sans extraction alcoolique } \\
\hline Orn & 4,02 & Pro & 0,48 \\
\hline Val & 3,57 & Ala & 0,40 \\
\hline Ala & 3,53 & Ser & 0,40 \\
\hline Leu & 2,78 & Gly & 0,39 \\
\hline Glu & 1,78 & Glu & 0,38 \\
\hline Gly & 1,75 & Arg & 0,26 \\
\hline Phe & 1,61 & Lys & 0,25 \\
\hline Ile & 1,45 & Asp & 0,13 \\
\hline Lys & 0,83 & Val & 0,12 \\
\hline His & 0,62 & Met & 0,09 \\
\hline Thr & 0,42 & Orn & 0,08 \\
\hline Pro & 0,40 & Phe & 0,08 \\
\hline Met & 0,30 & Leu & 0,07 \\
\hline Cit & 0,19 & His & 0,07 \\
\hline Arg & $0, \mathbf{1 6}$ & Thr & 0,06 \\
\hline Cys & 0,14 & Tyr & 0,06 \\
\hline Abu & 0,13 & Ile & 0,05 \\
\hline Ser & 0,12 & & \\
\hline Asp & 0,08 & & \\
\hline $\begin{array}{l}\text { Acides aminés } \\
\text { totaux }\end{array}$ & 23,88 & $\begin{array}{l}\text { Acides aminés } \\
\text { totaux }\end{array}$ & 3,31 \\
\hline
\end{tabular}

Les valeurs présentées ont été obtenues après analyse du liquide qui a circulé dans la poche d'un lapin opéré. Elles expriment la quantité totale d'acides aminés recueillis en $\mu$ moles. L'extraction alcoolique permet de dégager des acides aminés libres inclus dans des structures cellulaires. Les acides aminés sont classés par ordre d'importance décroissante.

\section{Le métabolisme pariétal}

Les variations des acides aminés libres pariétaux nous renseignent sur le métabolisme intermédiaire. Par rapport au cæcum témoin, les variations au niveau de la paroi de la poche (tabl. 4) se résument en une augmentation d'un premier groupe constitué par tous les acides aminés qui sont le départ des voies de synthèse : acide glutamique, glycocolle, alanine, aspartique, ou de garage : proline, aminobutyrique, citrulline, et par la diminution d'un deuxième groupe réunissant tous ceux qui sont situés en fin de chaîne et dont la synthèse par les cellules est plus difficile ou impossible : arginine, cystine, histidine, isoleucine, leucine, phénylalanine, tyrosine, valine.

L'accumulation relative et quantitative des acides aminés réunis dans le premier groupe a probablement pour conséquence leur sécrétion vers la cavité digestive de la poche.

La diminution des acides aminés du second groupe peut être due à l'absence de l'apport par absorption puisque la poche ne contient plus de matériel digestif. Elle peut également s'expliquer par une utilisation rapide de ces acides aminés dans la synthèse des protéines pariétales. 
Il semble, en effet, que la synthèse protéique soit très active dans la paroi de la poche cæcale puisque malgré le handicap de l'opération, et 1'absence de matériel digestif dans sa cavité, la teneur en acides aminés totaux ne varie pas. Ce résultat est à rapprocher de ceux obtenus par SALACROuP et RAyNaud (I965) sur le cæcum de lapins soumis à une période de jeûne non hydrique de Io jours. Ces auteurs constatent que la teneur en azote total de la paroi cæcale ne varie pas, alors qu'elle diminue de 30 p. Ioo dans les autres régions intestinales : estomac, intestin grêle, appendice côlon.

Dans la paroi de la poche, la synthèse protéique paraît même exaltée puisque dans le sang qui vient de l'irriguer, l'augmentation des protéines totales est hautement significative.

Il nous paraît difficile de donner une explication physiologique de ce fait, avant de connaître la nature des protéines susceptibles d'apparaître dans le sang veineux de la poche.

\section{CONCLUSION}

L'étude comparée de la poche et du cæcum témoin montre une similitude dans les variations de certains éléments plasmatiques, en particulier la paroi des deux parties puise dans le sang artériel la même quantité de phosphore inorganique et d'urée. Cette baisse de concentration de l'urée traduit probablement une excrétion cæcale semblable à celle qui a été montrée dans le cæcum du rat (COMBE et al., I965).

Les différences observées quant au calcium sérique, s'expliquent si l'on admet une absorption au niveau du cæcum témoin, qui au contraire de la poche, continue à recevoir dans sa cavité un matériel digestif, riche en débris végétaux et microorganismes, et contenant le calcium susceptible d'être absorbé.

Le problème se pose de savoir si le métabolisme pariétal du cæcum opéré est susceptible de traduire le métabolisme pariétal du cæcum normal. Le rapprochement entre l'activité protéosynthétique de la poche et celle du cæcum normal d'un lapin soumis au jeûne permet de penser qu'au moins sur le plan azoté, les mécanismes mis en jeu ne diffèrent guère. Le facteur déclenchant est manifestement lié à la raréfaction ou à l'absence du contenu dans la cavité digestive.

Reçu pour publication en mai 1975.

\section{SUMMARY}

\section{AMINO ACID METABOLISM IN THE WALL, OF AN ISOLATED}

CEACAL SAC IN RABBIT

Using a surgical technique, a sac is made with the distal part of the caecum; this is isolated from the proximal part which remains connected with the rest of the digestive tract.

The physiological functioning of the operated caecum is tested by observing variations of some blood components before and after irrigation of the parietal tissues. The concentration and distribution of total amino acids give an overall description of nitrogen composition, while variations in free amino acids characterize intermediary protein metabolism.

That the caecal sac has retained its physiological function as an organ is attested to by the homogeneity of values and coherence of variations; it seems to have strong proteosynthisizing activity. 


\section{RÉFÉRENCES BIBLIOGRAPHIQUES}

Beauville M., Raynaud P., Vernay M., I974. Concentration des acides gras volatils plasmatiques chez le Lapin. Ann. Rech. vétér., 5, 407-4II.

Bjornhag G., 1972. Separation and delay of contents in the Rabbit colon. Swedish J. agric. Res, 2, I $25-\mathrm{I} 36$.

Bonnafous R., Raynaud P., r968. Mise en évidence d'une activité lysante du côlon proximal sur les micro-organismes du tube digestif du Lapin. Arch. Sc. Physiol., 22, 57-64.

Bonnafous R., Raynaud P., I97o. Recherche sur les variations de la densité des micro-organismes dans le côlon du Lapin domestique. Experientia, 26, 52.

Carles J., Abravanel G., r97o. Dosage automatique et simultané des acides aminés et des guanidines. Bull. Soc. Chim. Biol, 52, 453-454.

Catala J., I972. Quelques modifications nutritionnelles et digestives consécutives à la suppression de la sécrétion pancréatique exocrine chez le Lapin. Thèse de Spécialité, Toulouse.

Combe E., Penot E., Charlier H., Sacouet E., I965. Métabolisme du Rat " germ-free ". Teneurs des contenus digestifs en certains composants azotés, en sodium et en potassium. Teneurs de quelques tissus en acides nucléiques. Ann. Biol. Anim. Bioch. Bioph., 5, I89-2o6.

Griffiths M., Davies D., I963. The role of soft pellets in the production of lactic acid in the Rabbit stomach. J. Nutr., 80, I 7I-I80.

McMillan R. T., Edwards W. A., Parker D. S., 1975. Observations on the volatile fatty acids present in the hind-gut and in the blood of the domestic Rabbit. Proc. Nutr. Soc., 34, 2 I A.

Marty J., Carles J., I968. Les acides aminés dans le cacum du Lapin. C. R. Acad. Sc. Paris, 267, $638-64 \mathrm{I}$.

Marty J., i973. Sur le rôle de la paroi cæcale du Lapin dans la régulation de l'aminoacidémie. C. $R$. Acad. Sc. Paris, 276, 569-571.

Marty J., Raynaud P., Carles J., I973. Les acides aminés et les acides carboxyliques dans le cæcum du Lapin. Ann. Biol. anim, Bioch. Biophys., 13, 429-45I.

Marty J., Carles J., 1973. Absorption des acides carboxyliques par la paroi cæcale du Lapin. Ann. Biol. anim. Bioch. Biophys., 13, 453-464.

Neill D. W., Doggart J. R., Mitchel L., 1967. Experience in the use of the SMA-I2 Analytical system in the Biochemistry laboratory. In: Technicon Symposia, Automation in analytical chemistry, 2.

Proto V., Cioffi L. A., I973. La ciecoectomia e la composizione aminoacidica di feci e ciecotrofo di Coniglio alimentati con due differenti diete. Boll. Soc. It. Biol. Sper., 49, I404-I4Io.

Salacroup J., Raynaud P., r 965 . Influence du jeûne sur la teneur en azote de la paroi du tractus digestif du Lapin. Arch. Sc. Physiol., 19, 19-28. 